\title{
A Multidimensional Walking Aid for Visually Impaired Using Ultrasonic Sensors Network with Voice Guidance
}

\author{
Olakanmi O. Oladayo \\ Electrical and Electronic Engineering, Technology Drive, Office 6, New Faculty of Engineering Building, University of \\ Ibadan, Ibadan, Nigeria \\ Email: olakanmi.oladayo@ui.edu.ng
}

\begin{abstract}
The application of engineering practices in medicine has immensely contributed to the recent findings in biomedical research areas. One of the products of this application is the development of sophisticated aids for physically challenged people. In this paper, visually impaired walking aid is designed and implemented using a network of ultrasonic sensors, thereby capable of detecting the direction and position of obstacle(s). The performance and functionality are also improved by the addition of alert light, and voice guidance signal which is relayed to a miniature headset. The recorded voice alerts the user of the presence and direction of the obstacle(s). The prototype of the multidimensional walking aid was able to detect obstacles within the range of $0 \mathrm{~m}$ to $1 \mathrm{~m}$ at the left, right and front of the stick with an appropriate voice alert. The test results of the prototype showed that the stick can efficiently guide its user.
\end{abstract}

Index Terms - Ultrasonic Sensor, Visual Impairment, Medical Equipment, Walking Aids, Embedded System

\section{INT RODUCTION}

Technology is the making, modification, usage and knowledge of tools, machines, techniques, crafts, systems, methods of organization in order to solve a problem, improve a preexisting solution to the problem, achieve a goal or perform a specific function. It can also be referred to the collection of such tools, machinery, modifications, arrangements and procedures. Technologies significantly affect human as well as other animal species' ability to control and adapt to their natural environments. The term can either be applied generally or to specific areas: examples include construction technology, medical technology, and information technology. Visual impairment is the loss of vision (of a person) or a significant limitation of visual capability resulting from either disease, trauma, or congenital or degenerative conditions that cannot be corrected by conventional means, such as refractive correction, medication, or surgery [1]. The following terms are used to describe the different levels of visual impairment: Partially sighted which occurs as a result of some type of $v$ isual problems. Low vision generally refers to a severe visual impairment, not necessarily limited to distance vision. Low vision applies to all individuals who are unable to read the newspaper at a normal viewing distance, even with the aid of eyeglasses or contact lenses. This can also be divided into two levels: Myopic - unable to see distant objects clearly, commonly called nearsighted or shortsighted, Hyperopic - unable to see close objects clearly, commonly called far-sighted or long-sighted. Blindness is a total or partial inability to see due to some physiological or neurological deficits. The levels of blindness are scaled according to the extent of vision loss. A situation where one is unable to see forms or sense light is referred to as total blindness. The following are a few eye disorders which can lead to visual impairments; retinal degeneration, albinism, cataracts, and glaucoma, muscular problems that result in visual disturbances, corneal disorders, diabetic retinopathy, congenital disorders, and infection. For a visually impaired person to navigate an environment without human assistance, the person must have adequate information about the travel path and also be able to detect obstacles within his/her navigation range. Without any form of assistance, most visually impaired people have to stress their other senses mostly the ears in an attempt to detect any possible obstacles in their path.

Visually impaired individuals have a very important goal of moving through unfamiliar spaces or situations without any form of aid. For easy movement in both familiar and unfamiliar environments, there are various methods devised to aid visually impaired individuals, examples of these methods are the use of walking canes, use of guide dogs which assist in navigation or by the help of another hu man with perfect sight. However, some of these methods have their shortcomings in terms of their efficiency. For example, the ordinary walking stick is unable to detect obstacles in its path except by direct contact with the obstacle. Also, the use of guide dogs is limited to environments the dog has been trained to navigate and any deviation from this trained environment will be dangerous to the individual. Hu man guides can be a letdown at times because the guide might not be available at the time his help is needed. This impedes the movement of the visually impaired person. All these shortcomings of some of the existing aids are the reason for developing an electronic walking aid that can help the visually impaired to navigate any environment without straining their other sense organs or depend on human guide. This is achieved through implementation of design 
which is capable of detecting obstacles in the navigation path and indicates the direction of the obstacles to prevent the user of the walking aid from coming in contact with the obstacles and hence preventing accidents.

The remaining part of this paper is arranged as follows: section 2 contains reviews of some related works on visually impaired walking aids. The design principle of the multidimensional walking aid is explained in section 3. Section 4 contains the construction and test details of the walking aid. Section 5 is the conclusion and recommendation.

\section{REVIEW OF RELATED WORKS}

Over the years, there has been an evolution of various methods of guiding blind people in moving around their environment without guidance. Some of the methods used over the years include the use of guide dogs; a small number of people emp loy guide dogs to assist in mobility. These dogs are trained to navigate around various obstacles, and to indicate when it becomes necessary to go up or down a step. The human half of the guide dog team does the directing, based upon skills acquired through previous navigation exercises. In this sense, the handler of the dog can be likened to an aircraft's navigator, who must know how to move from one place to another and the dog to the pilot, who guides them there safely

Another example of visually impaired navigation aid is non-electronic walking stick which has been in existence over a long period of time. There are different kinds of non-electronic walking sticks: traditional white cane also known as the Hoover cane is designed primarily to function as a navigation tool to detect obstacles in the path of the user. Its length usually depends on the height of the user and usually is as high as the chest of the user from the ground. There is also the guide cane which is shorter than the long cane, generally extending from the floor to the user's waist with more limited mobility function compared to the guide cane. It is usually used to scan for obstructions such as steps, curbs and ditches. Other types of cane include the identification cane; which has no mobility function, whose primary aim is to alert others about the visual impairment of the user, there is also the support cane which is to offer physical stability to the user of the cane and lastly the kiddie cane which is of a s maller size than the others but offers guidance and identification to visually impaired children [2].

The use of Global Position System (GPS) based navigation aid is one of the modern navigation aids among visually impaired people. This usually involves the use of software with embedded maps which is interfaced with a voice device that helps in giving blind people directions around their immediate environment. A GPS navigation system is not a replacement for the other forms of navigation, but it is better if used with any of the previous methods. Example of GPS blind people navigation system is a patent in reference [3] called Magic Wand Virtual Walking Stick. It is fully selfcontained and has a user interface designed specifically for the visually impaired, thus eliminating the problems associated with the existing solutions. The patented Magic Wand consists of an integrated global satellite receiver and inertial measurement unit. This makes it know the exact location, altitude at any given moment and current orientation of obstacles. That is, once the Magic Wand is pre-loaded with map information of the street, position of potential obstacles and landmark information the device can be used to direct the blind to doorways, crosswalk buttons, staircases, and dangerous obstacles such as high curbs or heaves in the sidewalk. The device behaves like a GPS device on level ground, directing the visually impaired using verbal commands and tactile feedback toward a destination.

With the advancement in technology, the development of the electronic walking cane has made navigation for visually impaired people easier by increasing their independence and safety. In [4] an electronic walking stick prototype was designed and implemented. This walking stick is capable of detecting obstacles in every direction through the network of infrared sensors. However, the walking stick can only alert the user of the presence of obstacles through beeping and cannot specify the direction of the obstacle(s). Apart from these design flaws, the stick cannot be used in the dark due to the absence of the alerting light.

In [5] an intelligent stick with ultrasonic sensor was proposed. This stick through the ultrasonic sensor gathered information on the user's pathway. This information is used to generate an artificial intelligent which would be used to control the movement of the attached DC motor in order to avoid an obstacle. The user of the stick has little or no contribution to the navigation thereby makes the user look like a zo mbie and a brakeless truck-pushed; Few of visually impaired persons like this scenario. Reference [6] presented a GPS based guiding stick for the visually impaired. This system used proximity sensors, ultrasonic sensors, GPS module, stereo cameras and dual feedback system- auditory as well as vibratory circuit to locate obstacles and inform the user on the present and distance of the obstacles on the pathway.

In embedded system development distance, measurement is vital and has wide application especially in robotic movement. Reference [7] highlighted this and proposed a cost effective method of determining distance of an object(s) using ultra sonic sensor and microcontroller. This method can be used to determine the distance of an obstacle in the pathway, therefore, giving a blind person pre knowledge of the obstacle's distance.

Another smart walking stick which detects and warns the user was proposed in [8]. This is similar to the one proposed in this paper, it contains voice and vibration alert, however, it can not specify the actual direction of the obstacle(s). Also in [9], A rduino Uno was used to build a voice alert walking stick for visually impaired using three ultrasonic sensors. 


\section{DESIGN DET AILS OF THE MULTIDIMENSIONAL W ALKING AID}

The multidimensional walking aid schematic and circuit diagrams are illustrated in fig. 1 and 5 respectively. The design consists of three stages which are power, control and output stages. The relationship between the different parts and stages of the model are shown by the directions of the arrows in the block diagram. A $9 \mathrm{~V}$ battery is connected to a voltage regulator to reduce the voltage level to $5 \mathrm{~V}$ which is the voltage required to power the microcontroller, ultrasonic transmitter and receiver, the voice recording chip, light detection circuit and voice output amplifier. A $40 \mathrm{KHz}$ signal is sent out by the ultrasonic transmitter. This will be reflected back to the ultrasonic receiver in case there is an obstacle along the pathway of the stick, and this activates one of the input pin of the microcontroller. Once this happened, the microcontroller will consequently activate the voice recording microchip which then gives the relevant output via the speaker. However, in case of absence of the obstacle along the pathway nothing will be sent to the microcontroller pin. The model also consists of light detection circuit which functions as a result of varying resistance of the light dependent resistor (LDR). The major components used and the functionality of each stage of operation of the electronic multidimensional walking aid are explained in the next sections:

\section{A. Function of the major components}

1. ISD 2590 (Single-Chip, Multiple Messages, Voice Record/Playback Device)

The ISD2590 provide single-chip, high quality, record playback solutions for 90 seconds message applications. The CMOS device consists of an on-chip oscillator, automatic gain control, microphone pre-amplifier, automatic gain control, anti-aliasing filter, smoothing filter, speaker amplifier and high density multi-level storage array. The ISD2590 chip is made to be compatible with a micro-controller to permit addressing and comple $\mathrm{x}$ messaging. Zero power message storage is provided, and messages are stored in non-volatile memory storage cells. This chip allows voice and audio signals to be stored in their natural form, providing high quality voice reproduction.

2. Microcontroller

The PIC16F887 is one of the latest products from microchip. It features all the components which modern microcontrollers normally have. For its low price, wide range of application, high quality and easy availability, it is an ideal solution in applications such as the control of different processes in industry, machine control devices, measurement of different values etc. For these, PIC16F887 was used in building the prototype of the multidimensional walking aid.

3. Voltage Regulator

A voltage regulator (regulator), usually having three legs, converts varying input voltage and produces a constant regulated output voltage. They are available in a variety of outputs. The most common part numbers start with the numbers 78 or 79 and finish with two digits indicating the output voltage. The number 78 represents a positive voltage and 79 negative one. The $78 \mathrm{XX}$ series of voltage regulators are designed for positive input. And the $79 \mathrm{XX}$ series is designed for negative input. Examples of 5V DC regulator names are: LM7805 or MC7805, $5 \mathrm{~V}$ DC regulator names are: LM7905 or MC7905, 6V DC regulator names are: LM7806 or MC7806, and $-9 \mathrm{~V}$ DC regulator names are: LM7909 or MC7909.

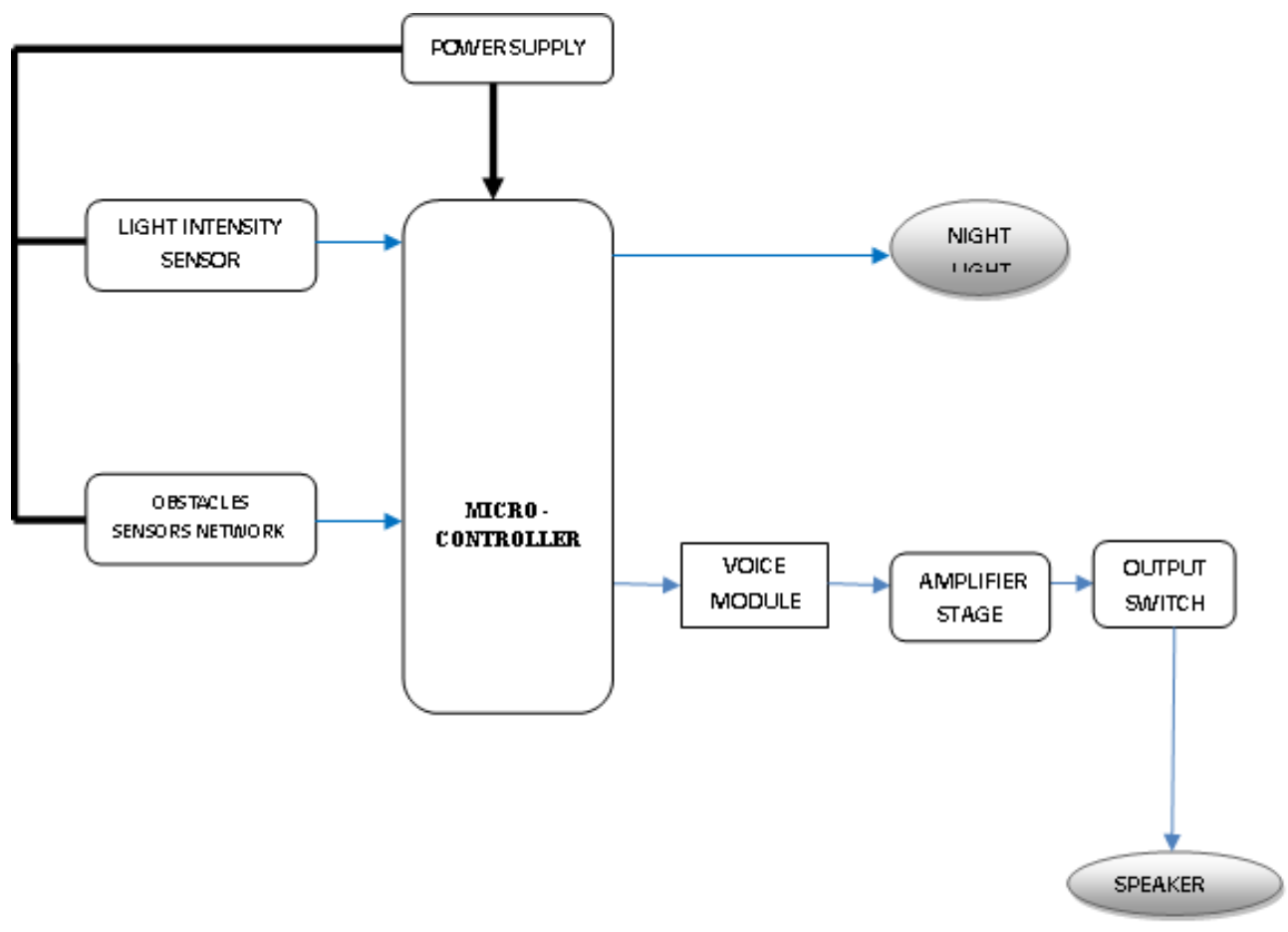

Fig. 1. Block diagram of the multidimensional walking aid 


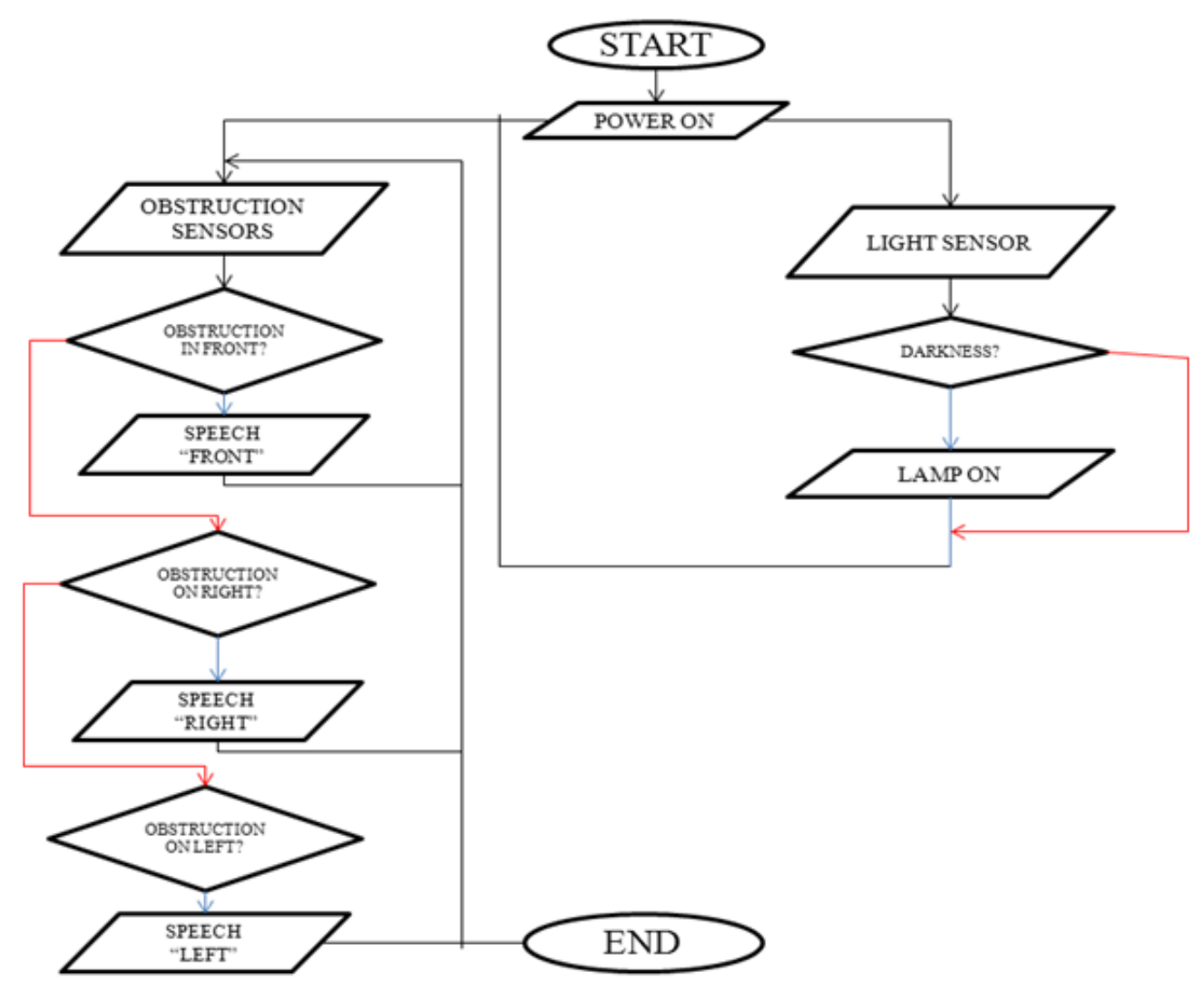

Fig. 2. Flowchart showing the working principle of the multidimensional walking aid

\section{Speaker}

This is the output device that gives the required speech when an obstacle is detected in the navigation path of the user of the walking stick or when help is required by the user of the cane. There are two types of speakers on this stick; the earphone that is constantly worn by the user, which gives the output when an obstacle is detected in the path of the user and the loudspeaker which also gives the voice output when configured to do so but mainly used to give a voice output (alert) in case of emergency such as when the walking stick falls down.

\section{B. Multidimensional stick stages}

The multidimensional walking stick design, as shown in the complete circuit diagram in fig. 5, consists of three functional stages, Function of each of these stages is explained below:

\section{Power Stage}

The power stage, as shown in fig. 3, supplies $5 \mathrm{~V}$ to power all the components in other stages. It contains a $9 \mathrm{~V}$ battery which is connected to a voltage regulator which regulates the voltage to $5 \mathrm{~V}$ which is the required voltage to power the ultrasonic sensors, mic rocontroller, ISD 2590 voice recording module, light detection circuit and LM386 amplifier. A resistor is connected to reduce the current entering the ultrasonic transmitter and receiver while a capacitor is connected to filter this voltage. The diode after the transmitting sensor ensures that the current does not flow back into the transmitting sensor. The $5 \mathrm{~V}$ enters the microcontroller (PIC 16F877A) v ia the positive supply pin.

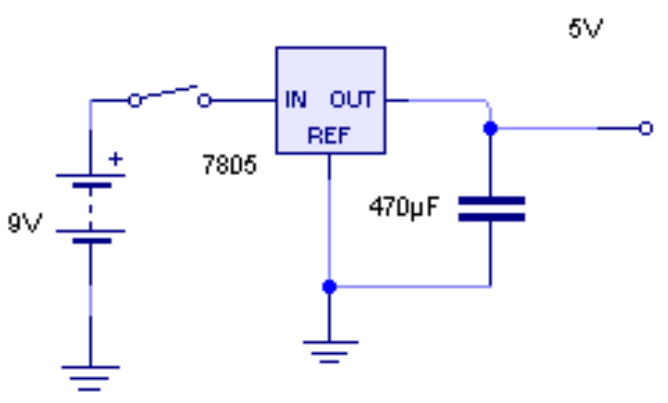

Fig. 3. Power stage of the multidimensional walking aid

\section{Control Stage}

The control stage involves the connection of the microcontroller, ultrasonic transmitters and receivers, 8 $\mathrm{MHz}$ crystal oscillator, and light detection circuit. The ultrasonic sensors and receivers operate at an ultrasonic frequency of $40 \mathrm{Khz}$; this $40 \mathrm{Khz}$ is sent out by the pin 17 of the PIC 16F877 microcontroller. Whenever obstacles are detected within the sensing range of the ultrasonic detectors, the $40 \mathrm{Khz}$ signal emitted is reflected by the obstacle back to the ultrasonic receiver which then sends a signal to any of pin 2, 3 or 4 . The crystal oscillator serves as the system clock which is a resonator (provides a precise resonance frequency of $8 \mathrm{MHz}$ ).

The light detection circu it consists of a light dependent resistor connected in a voltage divider arrangement with another resistor. The varying voltage of the LDR as a result of light intensity is used in the voltage divider arrangement to produce a biasing voltage to turn on the transistors which switch on the illumination LEDs 


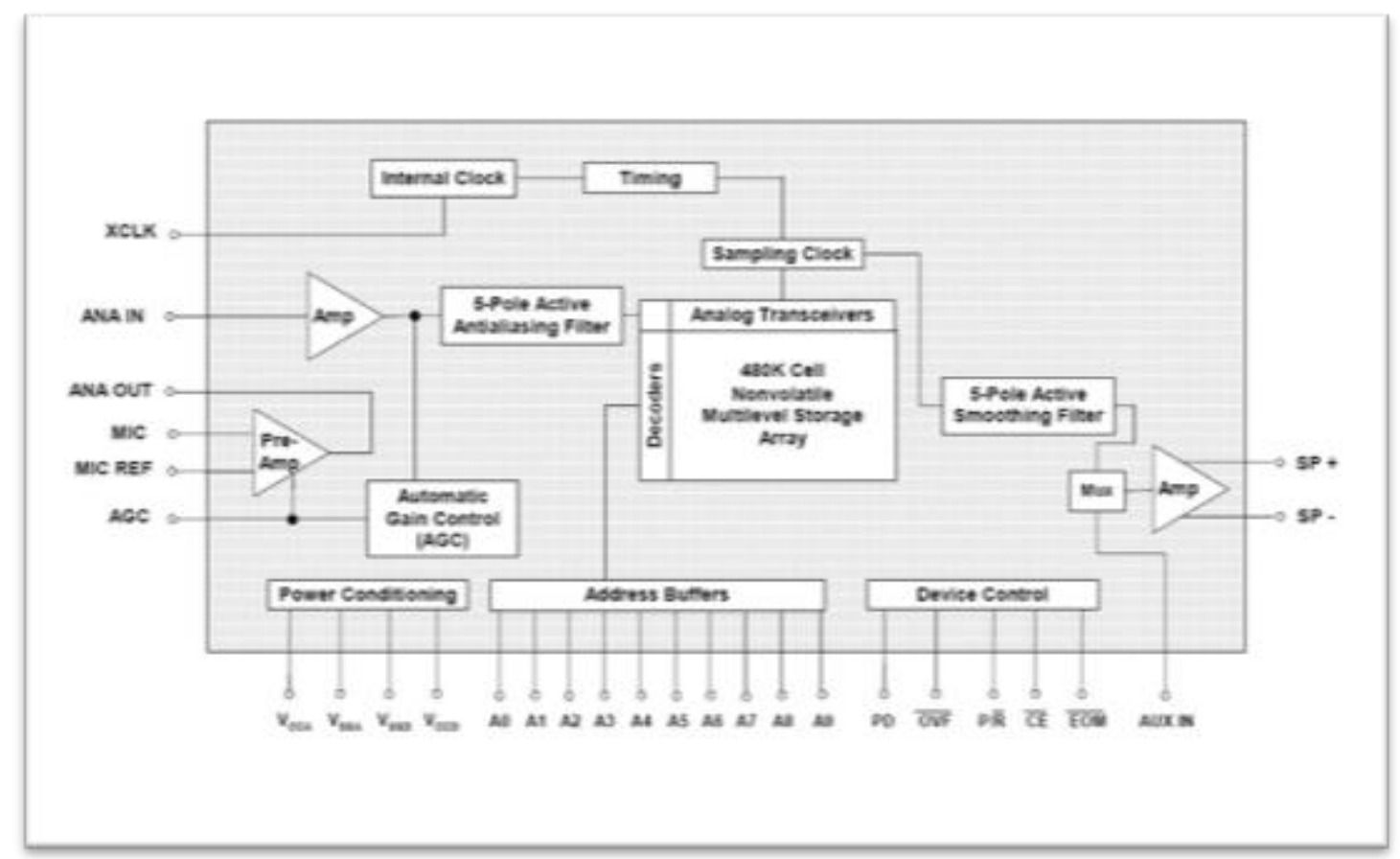

Fig. 4. Block diagram of ISD 2590 microchip

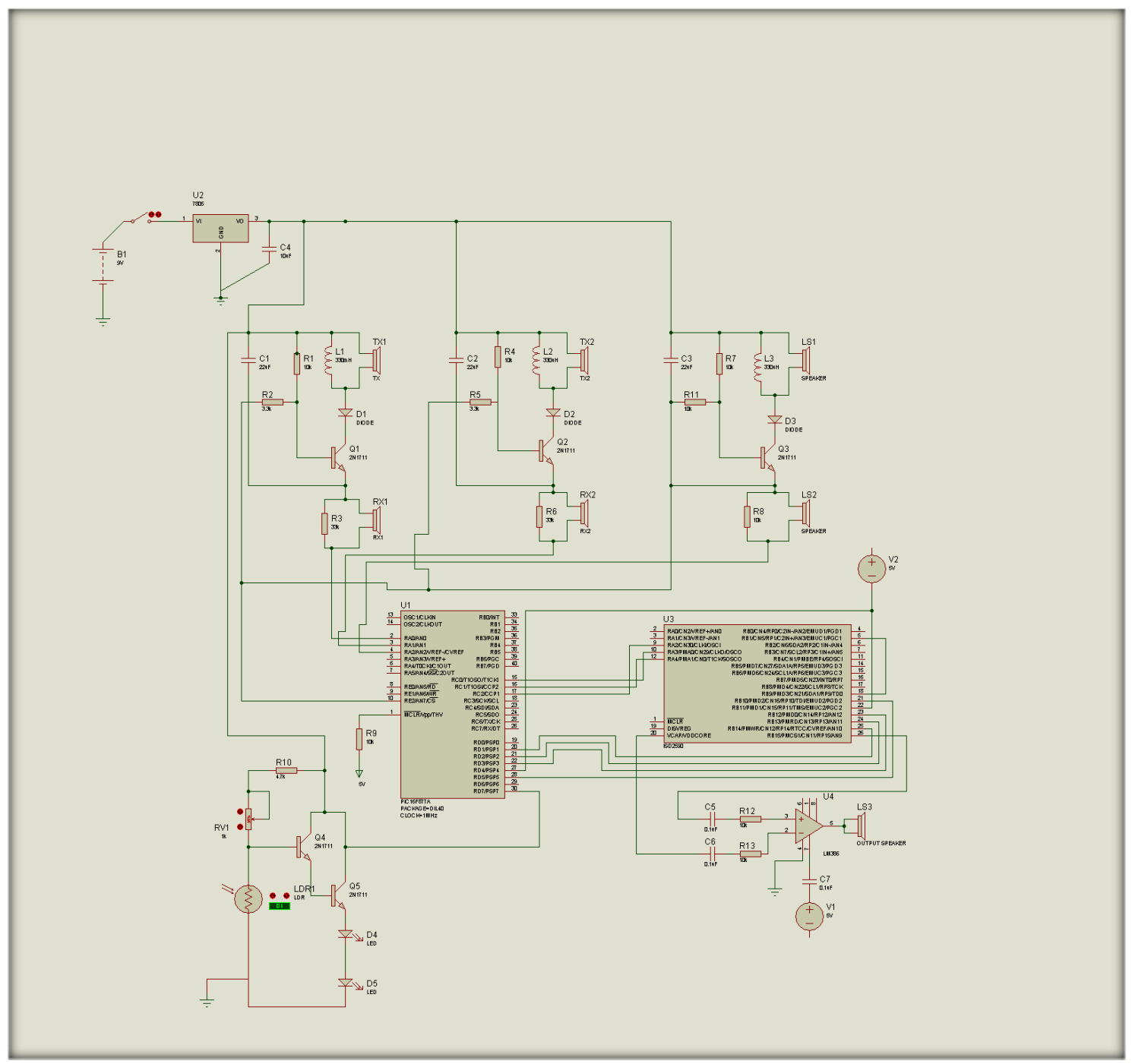

Fig. 5. Circuit diagram of the multidimensional walking aid 


\section{Output Stage}

The output stage involves interfacing the PIC 16F877A microcontroller with the ISD 2590 voice recording module and connecting it to the speaker to give a voice output whenever an obstacle is detected by any of the ultrasonic sensors on the three sides.

The microcontroller generates a $40 \mathrm{Khz}$ signal at the input stage which is sent out by the transmitting sensor of the ultrasonic sensor, this signal is reflected to the receiver of the sensor when the ultrasonic wave hits an obstacle within the range of the sensor. The signal received by the receiver is sent to the PIC $16 \mathrm{~F} 877 \mathrm{~A}$ microcontroller pins 2, 3, and 4.The state of each of these pins determines which of the recorded voice signals on ISD 2590 that will be played. The outputs of the ISD 2590 recording module are connected to an LM 386 amplifier. The LM 386 amplifies the received audio signal to make it clearer and more audible enough for reproduction via the speakers (earphone or outward speaker).

\section{CONSTRUCTION AND TESTING}

\section{A. Construction Detail}

As earlier stated, a $9 \mathrm{~V}$ was used to power the walking stick. The ultrasonic sensor consists of a trans mitter and receiver soldered side by side on a Vero-board. Three of this ultrasonic sensors are used, one for each of the three detection directions, these sensors are each connected to the power source in parallel connection. The PIC 16F877A, which serves as the control unit of the multidimensional walking stick, is powered by a $5 \mathrm{~V}$ power supply (output voltage of the 7805 regulator) via the pin 1 of the microcontroller. An $8 \mathrm{MHZ}$ crystal oscillator is connected to the microcontroller pins 12,13 and 14 to provide a clock signal input to the system. The output of the receiver of each of the ultrasonic sensors is connected to pin 2, 3 and 4 of the PIC 16F877A, which gives a corresponding output signal at the port pin via the output pins 33, 34 and 35 of the microcontroller. These output pins are connected to the pins 8, 9 and 10 of the ISD2590 voice recording module, these three address lines can be used to address eight memory spaces which are used to record the different messages that are to be used as a voice output signal.

The recording on the ISD 2590 voice recording module is done using a separate circuit consisting of a microphone connected to the ISD 2590. The messages are recorded in the eight addressable memory locations of the three output address lines. A marker is out at the end of each message to distinguish the end of one message to the start of the other. Control lines (pins 21-25) from the PIC 16F877A are connected to the pins 23-25 of the ISD 2590; these control lines specify the types of output that would be produced depending on the input signal received by the sensor and processed by the microcontroller. The output lines of the ISD 2590 are connected to the LM386 amplifier which amplifies the output signals from the ISD 2590. The speaker ports are connected to the output of the amplifier to give the required voice output. A light detection circuit consisting of a light dependent resistor and light emitting diodes is connected to the microcontroller to give the required illumination which serves as the alerting light to incoming people.

\section{B. Testing Detail}

The following tests were carried out to determine the different responses of the components that make up the multi-dimensional walking stick.

\section{Obstruction Detection Test}

With the ultrasonic transmitter (TX) fixed on the model board and the ultrasonic receiver (RX), the microcontroller is connected along with an LCD screen and LED to indicate the distance and presence of an obstacle respectively if any is detected. These components are powered from the $5 \mathrm{~V}$ supply of the voltage regulator. An obstacle was put in front of the sensor, and it was observed that the LED turned on when the obstacle was put in front. It was also observed that as the obstacle distance from the sensor was varied, the LCD displayed the appropriate distance depending on the distance (within a range of $<1 \mathrm{~m}$ ).

2. Voice Signal Output Test

The ISD 2590 is connected to a recorder module; the recorder consists of a microphone and the voice storage module. Random voice recordings are recorded onto the module via the microphone, the ISD 2590 voice storage module is then connected to the microprocessor on a via model board. When the appropriate signal is sent out from the microprocessor to the input of the ISD 2590 microchip, the corresponding output signal is produced via the speaker connected to the recording module.

3. Light Detection Test

The light dependent resistor is connected to a $100 \mathrm{~K}$ resistor in a voltage divider network. The LDR is a variable resistor that has high resistance in low light conditions and low resistance in well lit situations. The LEDs are connected to a transistor that is switched on when the voltage divider network (LDR included) generates the voltage required to turn on the LEDs. This circuit arrangement was taken to a dark room with low light intensity, and it was observed that the LEDs were turned on and when the fluorescent tubes of the room were turned on, the LEDs turned off. This indicates that that the LEDs are turned on based on the intensity of light detected by the LDR circuit.

\section{CONCLUSIONS \& RECOMMENDATIONS}

The design and construction of a multidimensional blind man's walking stick using ultrasonic sensors with voice output is of great benefit to blind people when it comes to independent mobility. It basically involves construction of the hardware, recording of the needed voice signals on the voice recorder and playback module and software programming of the microcontroller.

A voltage source of $9 \mathrm{~V}$ is connected to a voltage regulator to reduce the voltage to $5 \mathrm{~V}$ required to power the components. The microcontroller, ultrasonic 
transmitters and receivers, the voice recording module, the light detection circuit and the output amplifier are powered by the regulated 5V.The microcontroller sends a $40 \mathrm{KHz}$ pulse to the ultrasonic transmitter which sends out a signal to the environment, the receiver then receives the signal that is reflected by the obstacle in the pathway of the stick. The receiver sends a signal to the microcontroller which sends the appropriate signal to the voice recording module which gives the required output via the speaker.

The prototype of the visually impaired aid is able to detect obstacles in all the directions of the user. And, it is therefore capable of guid ing a visually impaired person to navigating his environment. However, the walking stick can not determine the distance of the obstacle to the multidimensional. This can be an improvement on this multidimensional stick.

\section{ACKNOWLEDGEMENTS}

Appreciation goes to the Electrical and Electronic Engineering Dept, University of Ibadan for providing all the necessary equipment for the implementation of the multidimensional walking stick. Also, the implementation was achieved with the help of my project students Omolayo A and Quadri Oladimeji

\section{REFERENCES}

[1] Arditi, A and Rosenthal, B. (1998) .Developing an Objective Definition of Visual Impairment. Proceedings of the International Low Vision . pp. Pp 331-334.

[2] Nicholas, Allan1995. Why Use the long Cane. 1st Edition. s.l. : Dexter,

[3] Virtual Walking Stick for the Visually Impaired. [Online] 2010. http://contest.techbriefs.com/2012/entries/medical/2746.

[4] Omolayo, A (2011). Design and Construction of a Multidimensional Sensor Blind Man Stick. Electrical and Electronic En gineering, University of Ibadan.. B.Sc Project.

[5] Kang, Sung Jae, Young Ho, Kim and Moon, In Hyuk (2001) Development of a mechatronic blind stick.. Seoul, Korea: s.n., 2001. Proceedings of the 2001 IEEE International Conference on Robotics \& Automation. pp. Pp 3209-3213.

[6] Dambhare, Shruti and A.Sakhare. (2011) .Smart stick for Blind: Obstacle Detection, Artificial vision and Real-time assistance via GPS . 2nd National Conference on Information and Communication Technology (NCICT) . pp. Pp 31-33.

[7] Shrivastava, K., Verma, A. and Singh, S. P. (2010) Distance Measurement of an Object or Obstacle by Ultrasound Sensors using P89C51RD2 ., International Journal of Computer Theory and Engineering,, Vol. 2.

[8] Wahab, Mohd Helmy Abd, et al. (2011), Smart Cane: Assistive Cane for Visually-impaired People. IJCSI International Journal of Computer Science Issues, Vol. 8.

[9] DIVYA, SRIRAMA, et al. (2010) Ultrasonic and voice based walking stick for the blind. Department of Electronics and Communication Engineering, Gokaraju Rangaraju Institute of Engineering and Technology.
[10] Novelline, Robert. (1997) Squire's Fundamentals of Radiology. 5th Edition. s.l. : Harvard University Press,. pp. Pp. 34-35. ISBNO-674-83339-2.

[11] Bruno, Pollet. (2012). Power Ultrasound in Electrochemistry: From Versatile Laboratory Tool to Engineering Solution. : John Wiley Sons, 2 ISBN1119967864.

[12] Corso, J. (1963) Bone-Conduction Thresholds for Sonic and Ultrasonic Frequencies., Journal of the Acoustical Society of America, Vol. 35.

[13] Takeda, S, et al. 5, (1992). Age Variation in the Upper Limit of Hearing., European Journal of Applied Phy siology, Vol. 65.

[14] Art, Popper and Richard, R, Fay. (1995) Hearing by Bats Handbook of Auditory Research. : Springer,

[15] Belote, Larry (2006). Low Vision Education and Training: Defining the Boundaries of Low Vision Patients. A Personal Guide to the VA Visual Impairment Services Program..

[16] DIVYA, SRIRAMA, et al (2010). Ultrasonic And Voice Based Walking Stick for the Blind. Gokaraju Rangaraju Institute Of Engineering And Technology. 2010.

\section{Author's Profile}

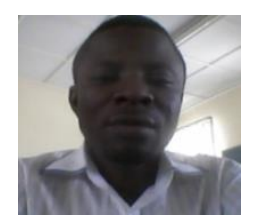

O.O Olakanmi received B.Tech in Computer Engineering from Ladoke Akintola University of Technology, Ogbomoso 2000 and M.sc in Computer Science from University of Ibadan, Ibadan. $\mathrm{He}$ is a lecturer in the Department of Electrical \& Electronic Engineering, University of Ibadan and major in Computer Architecture and Data Security.

How to cite this paper: Olakanmi O. Oladayo,"A Multidimensional Walking Aid for Visually Impaired Using Ultrasonic Sensors Network with Voice Guidance", International Journal of Intelligent Systems and Applications(IJISA), vol.6, no.8, pp.53-59, 2014. DOI: 10.5815/ijisa.2014.08.06 